\title{
Requirements for data integration platforms in biomedical research networks: A reference model
}

Matthias Ganzinger, Petra Knaup

Biomedical research networks need to integrate research data among their members and with external partners. To support such data sharing activities, adequate information technology infrastructure is necessary. To facilitate the establishments of such an infrastructure, we developed a reference model for requirements. The reference model consists of five reference goals and 15 reference requirements. Using the Unified Modeling Language, the goals and requirements are set into relation to each other. In addition, all goals and requirements are described textually in tables. This reference model can be used by research networks as a basis for the resource efficient acquisition of their project specific requirements. Further, a concrete instance of the reference model is described for a research network on liver cancer. The reference model is transferred into a requirements model of the specific network. Based on this concrete requirements model, a serviceoriented information technology architecture is derived and also described in this paper. 


\section{Requirements for data integration \\ 3 platforms in biomedical research \\ ${ }_{4}$ networks: A reference model}

5 Matthias Ganzinger*, Petra Knaup

6 Institute of Medical Biometry and Informatics, Heidelberg University, Heidelberg, Germany

7 * Corresponding author:

8 Matthias Ganzinger

9 Heidelberg University

10 Institute of Medical Biometry and Informatics

11 Im Neuenheimer Feld 305

1269120 Heidelberg

13 Germany

14 Phone: +49 6221 56-5143

15 E-mail: matthias.ganzinger@med.uni-heidelberg.de

\section{Abstract}

17 Biomedical research networks need to integrate research data among their members and with external

18 partners. To support such data sharing activities, adequate information technology infrastructure is

19 necessary. To facilitate the establishments of such an infrastructure, we developed a reference model

20 for requirements. The reference model consists of five reference goals and 15 reference requirements.

21 Using the Unified Modeling Language, the goals and requirements are set into relation to each other. In

22 addition, all goals and requirements are described textually in tables. This reference model can be used

23 by research networks as a basis for the resource efficient acquisition of their project specific

24 requirements. Further, a concrete instance of the reference model is described for a research network

25 on liver cancer. The reference model is transferred into a requirements model of the specific network.

26 Based on this concrete requirements model, a service-oriented information technology architecture is

27 derived and also described in this paper.

\section{Introduction}

29 Current biomedical research is supported by modern biotechnological methods producing vast amounts

30 of data (Frey, Maojo \& Mitchell, 2007; Baker, 2010). In order to get a comprehensive picture of the

31 physiology and pathogenic processes of diseases, many facets of biological mechanisms need to be

32 examined. Contemporary research, e.g. investigating cancer, is a complex endeavor that can be

33 conducted most successfully when researchers of multiple disciplines cooperate and draw conclusions 
34 from comprehensive scientific data sets (Welsh, Jirotka \& Gavaghan, 2006; Mathew et al., 2007). As a

35 frequent measure to support cooperation, research networks sharing common resources are established.

36 To generate added value from such a network, all available scientific and clinical data should be 37 combined to facilitate a new, comprehensive perspective. This requires provision of adequate 38 information technology (IT) which is a challenge on all levels of biomedical research. For example, it 39 is inevitable for research networks to use an IT infrastructure for sharing data and findings in order to

40 leverage joint analyses. Data generated by biotechnological devices can only be evaluated thoroughly

41 by applying biostatistical methods with IT tools.

42 However, data structures are often heterogeneous, resulting in the need for a data integration process.

43 This process involves the harmonization of data structures by defining appropriate metadata (Cimino, 44 1998). Depending on the specific needs and data structures of the research network, often a non45 standard IT platform needs to be developed to meet the specific requirements. An important 46 requirement might be the protection of data in terms of security and privacy, especially when patient 47 data are involved.

48 In the German research network SFB/TRR77 - "Liver Cancer. From Molecular Pathogenesis to 49 Targeted Therapies" it was our task to explore the most appropriate IT-architecture for supporting 50 networked research (Woll, Manns \& Schirmacher, 2013). The research network consists of 22 projects 51 sharing common resources and research data. To provide this network with a data integration platform 52 we implemented a service-oriented architecture (SOA) (Taylor et al.; Papazoglou et al., 2008; Wei \& 53 Blake, 2010; Bosin, Dessì \& Pes, 2011). The IT system is based on the cancer Common Ontologic 54 Representation Environment Software Development Kit (caCORE SDK) components of the cancer 55 Biomedical Informatics Grid (caBIG) (Komatsoulis et al., 2008; Kunz, Lin \& Frey, 2009). The 56 resulting system is called pelican (platform enabling liver cancer networked research) (Ganzinger et 57 al., 2011). Transfer of these data sharing concepts to other networks investigating different disease 58 areas is possible.

59 We consider our research network as a typical example for a whole class of biomedical research 60 networks. To support this kind of projects, we provide a framework for the development of data 61 integration platforms for such projects. Specifically, we strive for the following two objectives:

62 Objective 1: Provide a reference model of requirements of biomedical research networks regarding an 63 IT platform for sharing and analyzing data.

64 Objective 2: Design a SOA of an IT platform for our research network on liver cancer. It should 65 implement the reference model for requirements. While this SOA is specific to this project, parts can 66 be reused by similar projects. 


\section{Methods}

68 For the design of a data integration platform it is important to first capture the requirements of the

69 system's intended users. To support this task, we developed a reference model for requirements. A

70 reference model is a generic model, which is valid not only for a specific research network, but for a

71 class of such organizations. For the development of the reference model, we used the research network

72 on liver cancer as primary source. These requirements were consolidated and abstracted to get a

73 generic model that can be applied to other research networks.

74 In a first step, a general understanding of the network's aims and tasks was acquired by analyzing written descriptions of the participating projects. In addition, questionnaires were sent to the principal investigators to capture the data types and data formats used within the projects. In a second step, projects were visited and their research subjects and processes were captured by interviewing project members.

79 For the reference model, we use the term goal to describe the highest level of requirements. This is in accordance with ISO/IEC/IEEE 24765 where goal is defined as "an intended outcome" (ISO/IEC/IEEE, 2011). In contrast, requirement is defined as "a condition or capability needed by a user to solve a problem or achieve an objective" (ISO/IEC/IEEE, 2011). In our reference model, each requirement was related to a goal, either directly or indirectly. Requirements were later on mapped to concrete functions in the resulting data integration system. On the other hand, goals were used to structure requirements and usually do not lead to a specific function of the system.

To provide a more detailed characterization of the goals, we provide a standardized table for each of them. It covers the reference number, name, description and weight of the goal. Table 1 shows the structure of such a table. The complete set of tables for all goals is available in the supplementing information S1.

The requirements are documented in the same way as goals are. Figure 1 shows a Unified Modeling Language (UML) diagram with all elements used for describing of both goals and their subordinated requirements (Object Management Group, 2012). As for the goals, we provide a set of tables with more detailed descriptions for all requirements in supplementing information S1. In total, we identified 15 requirements for the reference model.

The instantiation of the reference model for requirements to meet the needs of a specific research network provides the basis for the architecture of the desired data integration and its subsequent implementation. We provide a concrete instance of a reference model as well as the resulting ITarchitecture in this manuscript.

For the research described in this paper, ethics approval was not deemed necessary. This work involved no human subjects in the sense of medical research, as e.g. covered by the Declaration of Helsinki (World Medical Association, 2013). At no time patients were included for survey or interview. Data 
104 the questionnaire. No research was conducted outside Germany, the authors' country of residence. 105 However, in other countries the approval of an institutional review board or other authority might be necessary to apply the reference model.

\section{7}

108

109

110

111

\section{Reference Model}

113 The reference model for requirements covers five reference goals $(\mathrm{RG})$. An overview of the goals and 114

\section{Results}

In this section we first describe the reference model for requirements. Then, we show how a concrete model for requirements and an IT-architecture is derived from this reference model. The reference model for requirements is an abstract model and thus a universally usable artefact. It is mapped in several steps to the network specific system architecture. their relations is shown in Figure 2 by means of a UML requirements diagram. The reference goals are:

- Conduct research project (reference goal RG1): The ultimate goal of a research network is to fulfill the intended research tasks. This usually corresponds to the project specification of the funding organization.

- Answer research questions (reference goal RG2): Each research network has specific research questions it pursues to answer. These questions frame the core of the network and led to its establishment in the first place.

- Create, store, and retrieve data (reference goal RG3): Research networks need data to conduct the project. Thus, it is necessary to generate and handle them.

- Analyze data (reference goal RG4): To generate knowledge out of the data it is necessary to analyze them.

- Control data access and usage (reference goal RG5): Research networks need to protect their data. This includes the prevention of unauthorized access to protected data like patient data as well as aspects of intellectual property rights that need to be respected by authorized users as well.

These goals are ordered in a hierarchical structure: Goal RG1 acts as the root node, which has the two sub goals RG2 and RG3. Goal RG4 is subordinated to Goal RG2, whereas Goal RG5 is a sub goal of Goal RG3.

Each goal has several requirements. In total, the reference model contains 15 reference requirements (RR). A UML diagram with all reference goals and reference requirements is shown in Figure 3.

Reference requirements are associated with the reference goals as follows:

Goal 3 is associated with the reference requirements to create data (RRI) and to retrieve external data (RR2). These two requirements respect possible sources of data necessary for the research network. Reference requirement RR3, represent data, is further defined by its subordinate requirements define syntax (RR4), define data model (RR5), identify data (RR6), and define semantics (RR7). 
139 Goal 5, control data access and usage, has two aspects, which are represented by reference

140 requirements RR8 and RR9. RR8 requires the creator's contribution in the generation of data for the 141 research network to be recognized when data are used by others. As a consequence, even users with 142 legitimate access to the system have to adhere to usage regulations. These regulations should be 143 checked and enforced by the system as far as possible. In contrast, RR9 covers the requirement to 144 protect data from unauthorized access.

145 The second group of reference requirements covers data analysis. At the highest level we identify goal 1462 , answer research questions. It is associated downstream with goal RG4, analyze data. Goal RG4 is 147 composed of two reference requirements: RR11 integrate data and RR13 define analytical process. 148 RR11 is associated with RR3, since the technical provision of data within the research network is of great relevance for the integration of data. RR13 has subordinate requirement RR12, define analytical methods, which covers the low-level data analysis methods. RR14 and RR15 cover two distinct instances of RR13: RR14 describes static workflows with all process steps being fixed. In this case, the order of analytical steps and data sources used cannot be changed by the users of the system. In contrast, RR15 considers dynamic workflows, allowing users to compose analytical steps and data sources as needed. Since the type of data involved in a dynamic workflow is not known upfront, this reference requirement is more demanding in terms of semantic description of data sources. Precise annotation of data sources is necessary in order to perform automated transformations for matching different data fields.

RR13 is further associated with RR10, show results. RR10 covers the requirement to present the results of the analysis adequately. Thus, it partially fulfils goal 2, answer research questions.

The reference model for requirements is the basis for a network specific requirements model. We present an example for creating such a model and all following steps in the next section. All goals and requirements from the reference model are mapped to network specific instances. In this process, elements of the reference model are checked for their applicability to the specific research network. Further special requirements of the network are considered at this point as well.

The network specific requirements model is then mapped to system properties. These are qualities contributed to the system by different components. At first, we consider abstract components instead of specific products. For example, in a research network, reference requirement RR 1 create data might be mapped to a system property automated creation of data services. This property is then mapped to the specific component responsible for the implementation of this property.

In a second step, the abstract components are mapped to specific components in accordance with the research network's requirements. Specific components can be preexisting modules with a product character, software development frameworks providing specific functionality, or newly developed components. 
174 In a final modelling step a distribution model of the components is created. All components need to be 175 mapped to system resources down to the hardware level. Among others, the following aspects have to 176 be considered is this step:

177

- Security: Components with high security requirements should be isolated against other, less sensitive components and thus be run on a separate system node.

- Performance: All components must be distributed in a way that availability of sufficient system resources is ensured.

- Maintainability: To ensure that the possibly complex distributed system can be managed efficiently, components should be grouped together in a sensible way.

\section{Sample Application: pelican}

We now describe a sample application of our reference model within the research network SFB/TRR77 on liver cancer. Further, we describe two specifications for metadata we developed for the research network.

\section{Specific model for requirements}

In this section we summarize key requirements specific to our research network. The complete list of requirements is shown in supplementing information $\mathrm{S} 2$.

The first goal of the research network, an instance of reference goal RG1, is defined by its research assignment of gaining a deeper understanding of the molecular basis of liver cancer development. This spans research on the chronic liver disease to progression of metastatic cancer. Further, the research network aims to identify novel preventive, diagnostic and therapeutic approaches on liver cancer. Subordinated to goal G1 is G3, the instance of reference goal RG3 regarding the data necessary for the network. Since molecular processes play a major role within the research network, genomic microarray data are of central importance. They are complemented by imaging data like tissue microarray (TMA) data and clinical data.

Goal G2, answering research questions, is characterized by the following two questions:

- Which generic or specific mechanisms of chronic liver diseases, especially of chronic virus infections and inflammation mediated processes predispose or initiate liver cancer?

- Which molecular key events promoting or keeping up liver cancer could act as tumor markers or are promising targets for future therapeutic interventions?

Goal G5 requires making the data available for cross project analysis within the network, but to protect data against unauthorized access at the same time. Especially important to the members of the project is the requirement R8, subordinated to goal G5: The projects contributing data to the network require keeping control over the data in order to ensure proper crediting of their intellectual property. Thus, they require fine-grained rules for data access control. Depending on the type of data, they should be available only to specific members of the network, to all members of the project or the general public. 


\section{System architecture}

To acknowledge the project's requirement R8 to keep the ownership over their data, federation is the underlying concept of the system architecture. Technically, pelican implements a SOA. All data sources of the projects are transformed into data services and made available to the research network. The data services stay under the control of the contributing project. This can even go as far as running the service on computer hardware on the projects' premises. Data services are complemented by analytical services. All services are described by standardized metadata to help finding appropriate services and allow for automated access to the services' interfaces. Using a web-based user interface, researchers can chain data services and analytical services to answer specific research questions.

\section{Component model}

The requirements are mapped to system properties first. In the next step, components are identified to provide these properties as module of the new system. In Table 2 we show the complete chain of mappings from requirements over system features to components. Each component is realized either by a readily available product or by a newly developed module. In Table 3 we give an overview of our components.

The portal component provides the user interface to the system. It is implemented using the open source software Liferay (http://www.liferay.com, accessed: 2014-07-03) (Sezov, Jr., 2012). Liferay provides a number of functions affecting several components of our model. Thus, we provide a decomposition of the portal components in Figure 4. One important subcomponent of the portal is the document management system. It is realized by the Alfresco component (http://www.alfresco.com, accessed: 2014-07-03) (Berman, Barnett \& Mooney, 2012). The user interface of Alfresco can be integrated into the Liferay portal or be accessed with a separate unified resource locator (URL). The portal provides user management functionality to control access to portal pages and components like portlets (Java Community Process, 2008). However, the user account information including username, passwords, and others is stored in a separate component using the Lightweight Directory Access Protocol (LDAP). Thus, it is possible for all components of the SOA-network to commonly access the users' identity information.

Data services are generated by using caCORE SDK (Wiley \& Gagne, 2012). With caCORE SDK it is not necessary to program the software for the service in a traditional way. Instead, a UML data model in Extensible Markup Language Metadata Interchange (XMI) notation has to be prepared (Object Management Group, 2002; Bray et al., 2006). From this model, caCORE SDK generates several artefacts resulting in a deployment packages for Java application servers like apache tomcat (The Apache Software Foundation, 2014). To simplify this process for spreadsheet based microarray data, we developed software tool to generate the XMI file as well. As a result, a service conforming to the web services specification ready for deployment is generated. For the provision of network specific metadata we chose TemaTres to serve our controlled vocabulary in standard formats like SKOS or Dublin Core (Weibel, 1997; Miles \& Bechhofer, 2009; Gonzales-Aguilar, Ramírez-Posada \& Ferreyra, 2012). Our analytical services are backed by the open source language and environment for statistical 
247 computing called $R$ ( $R$ Core Team, 2014). $\mathrm{R}$ is integrated into the services using the Rserve component 248 (Urbanek, 2003).

\section{Deployment model}

250 In a final modelling step the components are distributed to the physical resources available for the

251 system. In our case we used two servers with a common virtualization layer based on VMware

252 VSphere server. Thus, all nodes in our deployment model represent virtual machines (VM). Using

253 virtual switches, routers, and firewall appliances we were able to implement our Internet Protocol (IP)

254 network infrastructure. To enhance security, we implement a network zoning model comprised of an

255 internet zone, a demilitarized zone (DMZ) and an internal zone. Figure 5 shows the deployment model

256 in UML notation. The services shown in the model (service 1 to service $n$ ) are to be considered as

257 examples, since the concrete number of services is permanently changing. The deployment model also

258 reflects the different levels of control that can be executed by the owners of the data. They range from

259 shared nodes on the common servers over a dedicated VM to deployment on external hardware

260 controlled by the respective projects.

\section{Discussion}

262 In this manuscript, we describe a reference model for the requirements of research networks towards an 263 IT platform. For many funding programs including research grants of the European Commission the 264 collaboration of several research organizations at different sites is mandatory. This leads to a structural 265 similarity to our research network on liver cancer. Even though other research networks will have 266 different research aims, there are still requirements that are common to most networks. Since the 267 reference model already covers a basic set of requirements, it allows future research networks to focus 268 on defining specific requirements distinguishing them from other networks.

269

270

271

Users of the reference model are responsible for assessing the reference model's applicability to their project-specific needs. The reference model is based on data of a real research network that were generalized. To avoid bias in the model that might hinder transferability, we incorporated different views in the process of constructing the model. However, the transferability of the model to another context is, as for any model, limited. As a consequence, future research networks will have to derive a project specific instance of the reference model to reflect the corresponding characteristics of the project. The reference model is a tool, intended to help its users to create a concrete model covering the requirements of a research network with a high degree of completeness. The reference model provides guidance for this task. We expect, it helps reducing the effort to acquire all requirements.

A common technique used in requirements engineering for software is use case modelling (Jacobson, 1993; Bittner \& Spence, 2003). A use case is a compact scenario describing certain aspects of how a technical system behaves and how it interacts with other actors like its users. A use case model is a way of capturing requirements in an interactive way. Thus, a use case model can be developed further into a requirements model. In our context we found it hard to apply the use case approach since the researchers in our network mostly have biomedical backgrounds and thus are not familiar with 
284

software development. Nevertheless, use case modelling might be a helpful tool for other research networks when applying our reference model.

We applied the reference model successfully to a research network on liver cancer. Some specific requirements in this network led to the decision to set up a federated system allowing for a maximum of control of the individual projects over their respective data. The system was implemented as a service-oriented architecture using, among others, components of the caBIG project. A public version of our system with limited functionality is available at https://livercancer.imbi.uni-heidelberg.de/data. There, a gene symbol like BRCA1 can be entered into the search field and services are invoked for data retrieval as discussed in this paper. Other projects can benefit from this architecture as well, but the architecture is tailored to research networks with the requirements of federating data as data services. With this architecture, we try to acknowledge the data protection requirements of the participating projects. Still, further research regarding the use of data and crediting creatorship of data is necessary. First steps were made as part of this project (He et al., 2013).

In case the requirements regarding data control are more relaxed, an alternative would be to keep the data in a central data warehouse instead of the federation. In that case, i2b2 might be a suitable component to provide the data warehouse component (Murphy et al., 2007). Such a centralized approach also affects, how and when data are harmonized: In a central research data warehouse data are harmonized at the time of loading the database which ideally leads to a completely and consistently harmonized data base. In a service-oriented approach data are provided by means of data services as they are. All services are described by corresponding metadata enabling automated transformation of the data at time of access.

Our sample research network concentrates more on basic research than clinical application. In the future, we plan to apply our reference model to further projects with a stronger translational component. By doing so, we will be able to reevaluate the framework in a more clinical context.

\section{References}

Baker M. 2010. Next-generation sequencing: adjusting to data overload. Nature Methods 7 (7):495-499.

Berman AE, Barnett WK, Mooney SD. 2012. Collaborative software for traditional and translational research. Human genomics 6:21.

Bittner K, Spence I. 2003. Use case modeling. Boston, Mass.: Addison-Wesley.

Bosin A, Dessì N, Pes B. 2011. Extending the SOA paradigm to e-Science environments. Future Generation Computer Systems 27 (1):20-31.

Bray T, Paoli J, Sperberg-McQueen CM, Maler E, Yergeau F, Cowan J. 2006. Extensible Markup Language (XML) 1.1 (Second Edition). World Wide Web Consortium. Available at http://www.w3.org/TR/xml11/(accessed 9 December 2014).

Cimino JJ. 1998. Desiderata for controlled medical vocabularies in the twenty-first century. Methods of information in medicine 37 (4-5):394-403. 
Frey LJ, Maojo V, Mitchell JA. 2007. Bioinformatics linkage of heterogeneous clinical and genomic information in support of personalized medicine. Yearbook of medical informatics:98-105.

Ganzinger M, Noack T, Diederichs S, Longerich T, Knaup P. 2011. Service oriented data integration for a biomedical research network. Studies in health technology and informatics 169:867-871.

Gonzales-Aguilar A, Ramírez-Posada M, Ferreyra D. 2012. TemaTres: software para gestionar tesauros. El Profesional de la Información 21 (3):319-325.

He S, Ganzinger M, Hurdle JF, Knaup P. 2013. Proposal for a data publication and citation framework when sharing biomedical research resources. Studies in health technology and informatics 192:1201.

ISO/IEC/IEEE. 2011. Systems and software engineering--vocabulary: Ingénierie des systèmes et du logiciel--vocabulaire. ISO/IEC/IEEE 24765. Geneva, Switzerland, New York: ISO/IEC; Institute of Electrical and Electronics Engineers.

Jacobson I. 1993. Object-oriented software engineering: A use case driven approach. Wokingham: Addison-Wesley.

Java Community Process. 2008. Java ${ }^{\mathrm{TM}}$ Portlet Specification Version 2.0. Available at http://www.jcp.org/en/jsr/detail?id=286 (accessed 9 December 2014).

Komatsoulis GA, Warzel DB, Hartel FW, Shanbhag K, Chilukuri R, Fragoso G, Coronado S de, Reeves DM, Hadfield JB, Ludet C, Covitz PA. 2008. caCORE version 3: Implementation of a model driven, service-oriented architecture for semantic interoperability. Journal of biomedical informatics 41 (1): 106-123.

Kunz I, Lin M, Frey L. 2009. Metadata mapping and reuse in caBIG ${ }^{\mathrm{TM}}$. BMC bioinformatics 10 (Suppl 2):S4.

Mathew JP, Taylor BS, Bader GD, Pyarajan S, Antoniotti M, Chinnaiyan AM, Sander C, Burakoff SJ, Mishra B. 2007. From Bytes to Bedside: Data Integration and Computational Biology for Translational Cancer Research. PLoS Computational Biology 3 (2):e12.

Miles A, Bechhofer S. 2009. SKOS simple knowledge organization system reference. World Wide Web Consortium. Available at http://www.w3.org/TR/skos-reference/ (accessed 9 December 2014).

Murphy SN, Mendis M, Hackett K, Kuttan R, Pan W, Phillips LC, Gainer V, Berkowicz D, Glaser JP, Kohane I, Chueh HC. 2007. Architecture of the open-source clinical research chart from Informatics for Integrating Biology and the Bedside. AMIA ... Annual Symposium proceedings / AMIA Symposium. AMIA Symposium:548-552.

Object Management Group. 2002. OMG XML Metadata Interchange (XMI) Specification: Version 1.2. Available at www.omg.org/cgi-bin/doc?formal/02-01-01.pdf (accessed 9 December 2014).

Object Management Group. 2012. Information technology - Object Management Group Unified Modeling Language (OMG UML), Infrastructure. Version 2.4.1. Available at http://www.omg.org/spec/UML/2.4.1/Infrastructure/PDF (accessed 9 December 2014).

Papazoglou MP, Traverso P, Dustdar S, Leymann F. 2008. Service-Oriented Computing: a Research Roadmap. Int J Coop Info Syst (International Journal of Cooperative Information Systems) 17 (02):223-255.

R Core Team. 2014. R: A Language and Environment for Statistical Computing. Sezov R, Jr. 2012. Liferay in action: The official guide to Liferay portal development. Shelter Island: Manning. 

International Conference on Scientific and Statistical Database Management, 2004, 443-444.

The Apache Software Foundation. 24.11.2014. Apache Tomcat - Welcome! Available at http://tomcat.apache.org/ (accessed 2 December 2014).

Urbanek S. 2003. Rserve: A Fast Way to Provide R Functionality to Applications. In: Hornik K, Leisch F, Zeileis A, eds. Proceedings of the 3rd International Workshop on Distributed Statistical Computing (DSC 2003). Wien.

Wei Y, Blake MB. 2010. Service-Oriented Computing and Cloud Computing: Challenges and Opportunities. IEEE Internet Computing 14 (6):72-75.

Weibel S. 1997. The Dublin Core: A Simple Content Description Model for Electronic Resources. Bulletin of the American Society for Information Science and Technology 24 (1):9-11.

Welsh E, Jirotka M, Gavaghan D. 2006. Post-genomic science: cross-disciplinary and largescale collaborative research and its organizational and technological challenges for the scientific research process. Philosophical Transactions of the Royal Society A: Mathematical, Physical and Engineering Sciences 364 (1843):1533-1549.

Wiley A, Gagne B. 2012. caCORE SDK Version 4.3 Object Relational Mapping Guide. Available at

https://wiki.nci.nih.gov/display/caCORE/caCORE + SDK +Version+4.3+Object+Relational $+M$ apping+Guide (accessed 9 December 2013).

Woll K, Manns M, Schirmacher P. 2013. Sonderforschungsbereich SFB/TRR77: Leberkrebs. Der Pathologe 34 (S2):232-234.

World Medical Association. 2013. World Medical Association Declaration of Helsinki: ethical principles for medical research involving human subjects. JAMA 310 (20):2191-2194. 
1

Overview of the UML elements used in requirements diagrams.

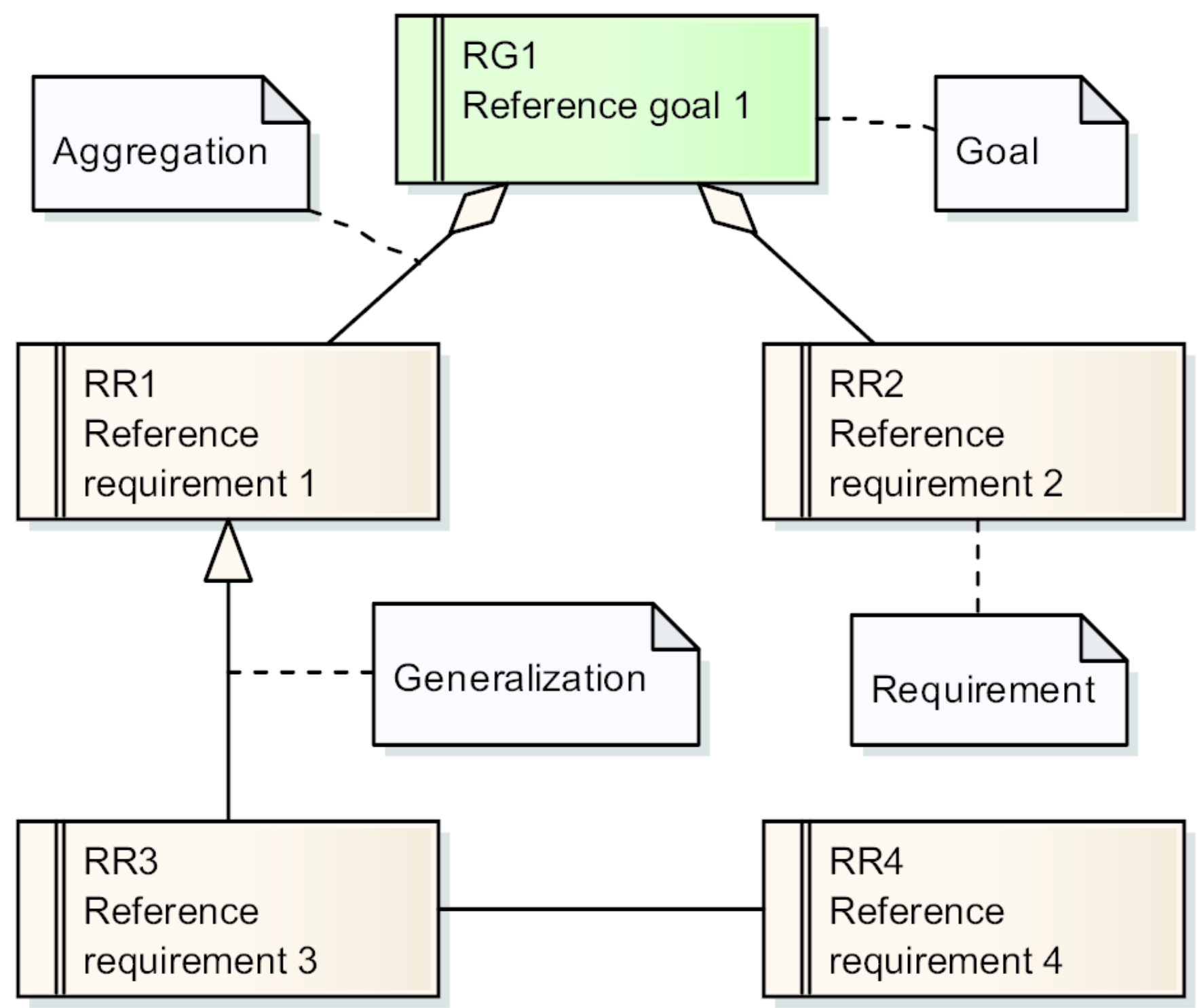


2

Reference model for goals of a research network.

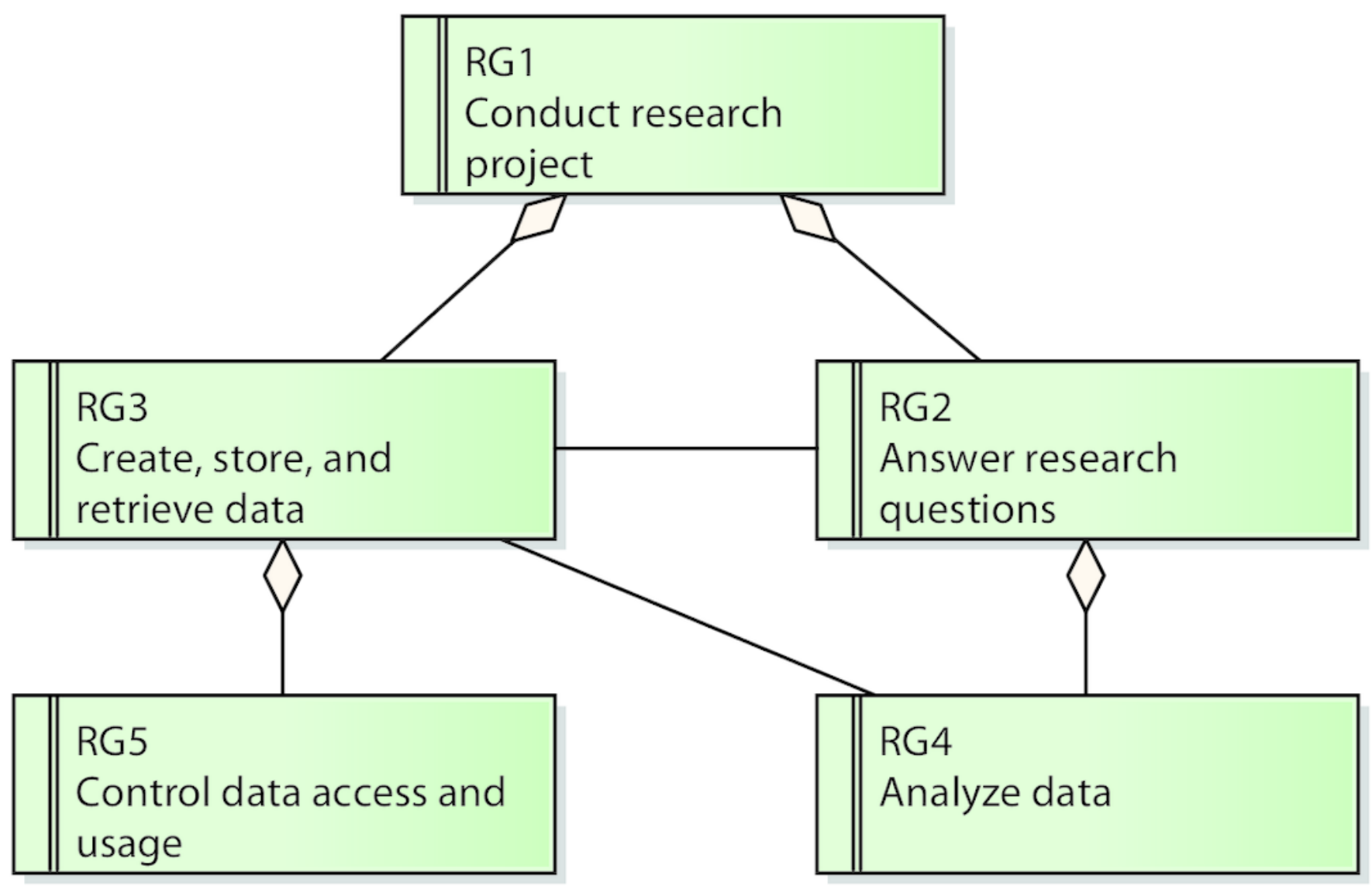


3

Reference model for goals and requirements of a research network.

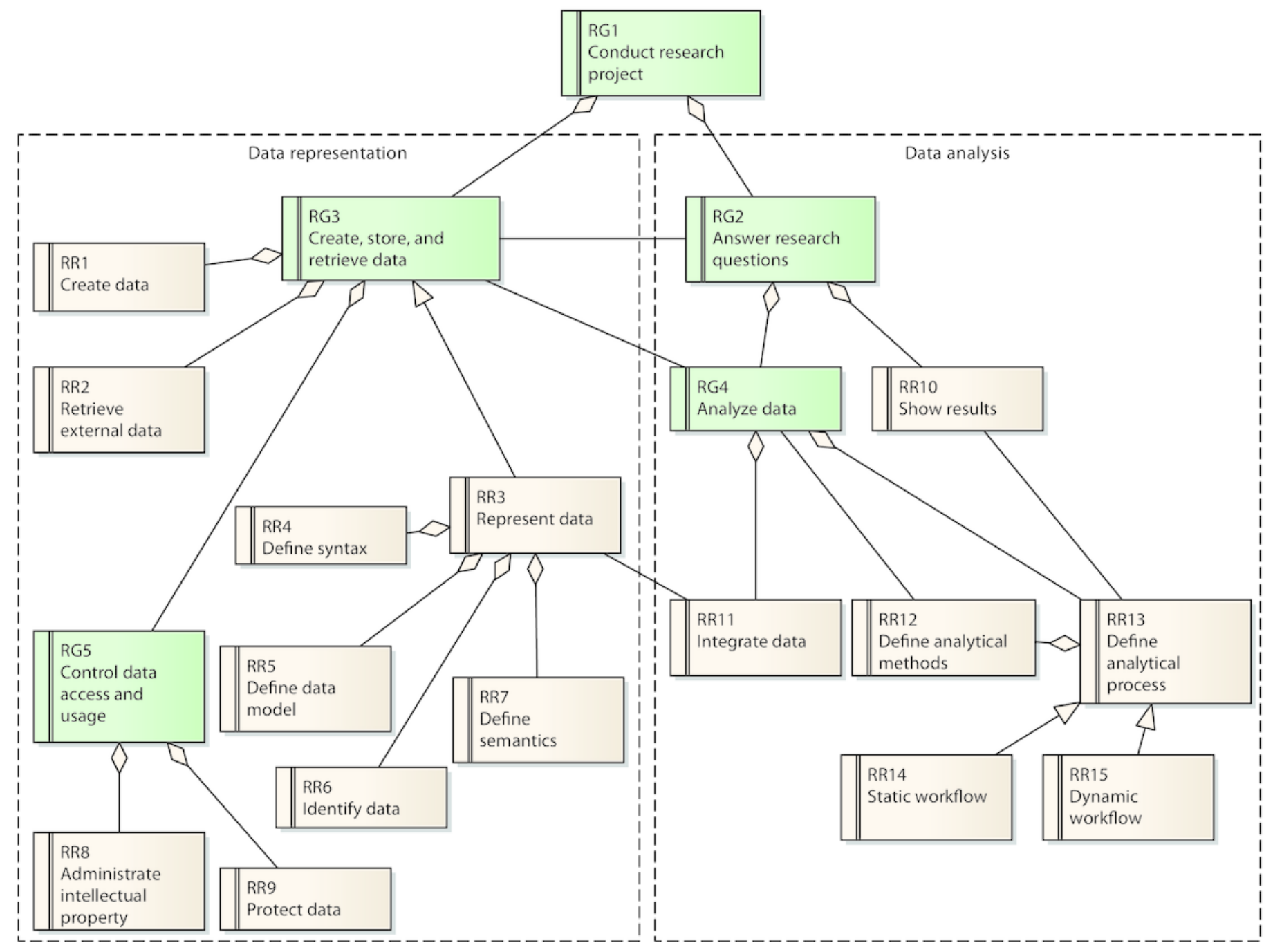


4

Structure of the component portal.

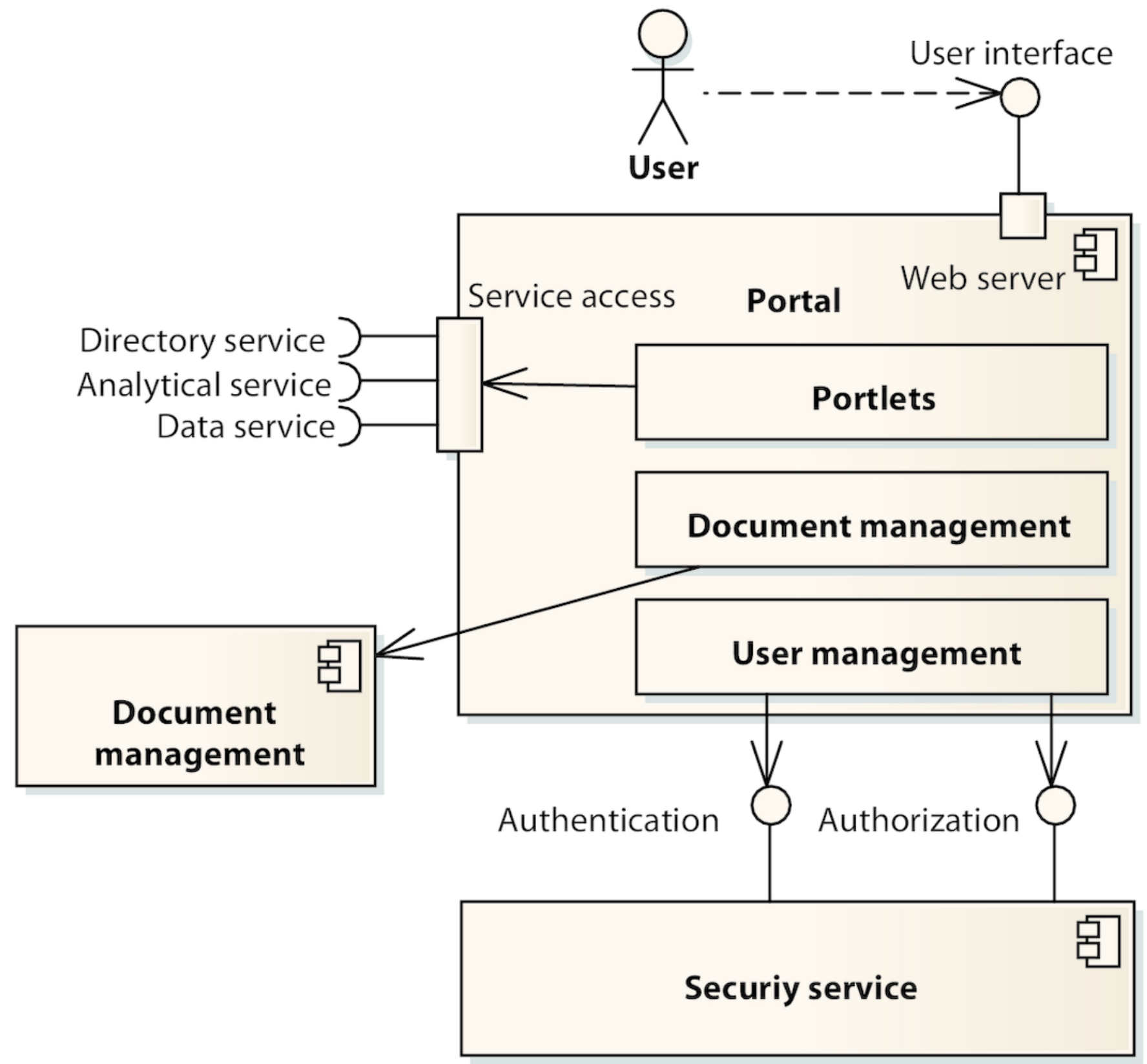


Deployment diagram of the components of the architecture in UML notation.

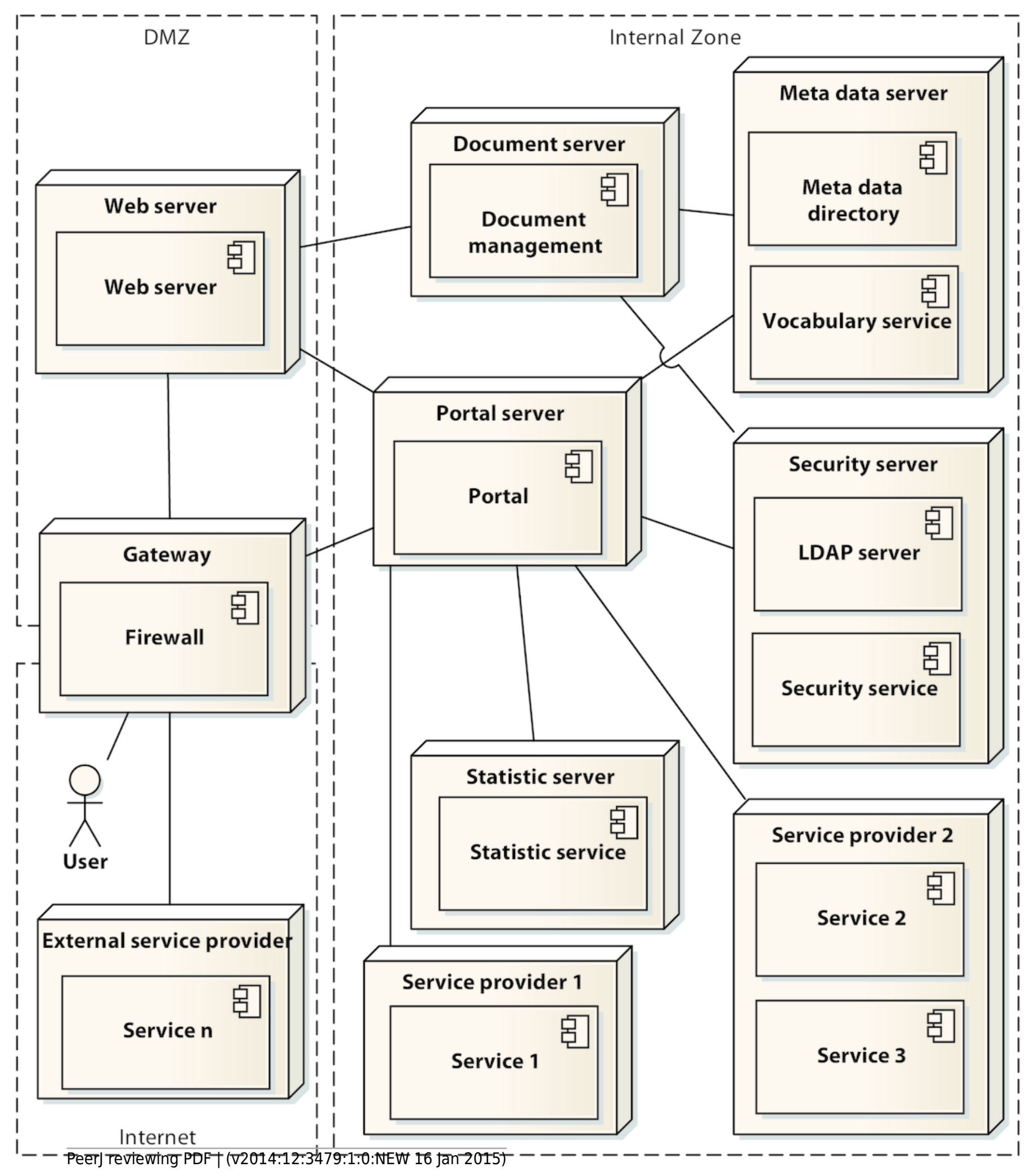




\section{Table $\mathbf{1}$ (on next page)}

Schema for documenting reference requirements and goals. 


\section{PeerJ Reviewing Manuscript}

\begin{tabular}{ll}
\hline Feature & Explanation \\
\hline Number & Number for uniquely identifying requirements \\
Name & Name of the requirement \\
Description & Verbal description of the requirement and its properties \\
Weighting & $\begin{array}{l}\text { Importance of the requirement for fulfilling the goals of the project } \\
\text { (low, medium, high) }\end{array}$ \\
\hline
\end{tabular}


Table 2 (on next page)

Mapping of requirements to corresponding system features and components. 


\section{PeerJ Reviewing Manuscript}

\begin{tabular}{|c|c|c|}
\hline Requirement & System feature & Component \\
\hline \multicolumn{3}{|c|}{ Requirements associated with G2 } \\
\hline R1 Create data & $\begin{array}{l}\text { Automated creation of data } \\
\text { services }\end{array}$ & Data service framework \\
\hline R2 Retrieve external data & $\begin{array}{l}\text { Integration of external data } \\
\text { services }\end{array}$ & $\begin{array}{l}\text { Data service framework, } \\
\text { portal }\end{array}$ \\
\hline R3 Represent data & $\begin{array}{l}\text { Data service, } \\
\text { document service }\end{array}$ & $\begin{array}{l}\text { Data service framework, } \\
\text { document management } \\
\text { system }\end{array}$ \\
\hline R4 Define syntax & Service description & Data service framework \\
\hline R5 Define data model & Defined data model & Data service framework \\
\hline R6 Identify data & $\begin{array}{l}\text { Provisioning of information } \\
\text { on service location }\end{array}$ & Meta data directory \\
\hline R7 Define semantics & $\begin{array}{l}\text { Definition of controlled } \\
\text { vocabulary and ontologies }\end{array}$ & Terminology server \\
\hline \multicolumn{3}{|c|}{ Requirements associated with G4 } \\
\hline $\begin{array}{l}\text { R8 Administrate } \\
\text { intellectual property }\end{array}$ & Log data usage & Portal, data service \\
\hline R9 Protect data & $\begin{array}{l}\text { User authentication } \\
\text { User authorization }\end{array}$ & $\begin{array}{l}\text { Portal, security service } \\
\text { Portal, data service, security } \\
\text { service }\end{array}$ \\
\hline \multicolumn{3}{|c|}{ Requirements associated with G5 } \\
\hline R10 Show results & Data specific portlets & Portal \\
\hline R11 Integrate data & Analytical services & $\begin{array}{l}\text { Portal, statistics service, Data } \\
\text { service framework }\end{array}$ \\
\hline $\begin{array}{l}\text { R12 Define analytical } \\
\text { methods }\end{array}$ & Statistical methods & Statistics service \\
\hline $\begin{array}{l}\text { R13 Define analytical } \\
\text { process }\end{array}$ & Documentation service & $\begin{array}{l}\text { Document management } \\
\text { system }\end{array}$ \\
\hline R14 Static Workflow & $\begin{array}{l}\text { Workflow in portal } \\
\text { application }\end{array}$ & Portal \\
\hline R15 Dynamic Workflow & Flexible pipeline & Pipeline management \\
\hline
\end{tabular}


Table 3(on next page)

Specification of concrete implementation components for the elements of the component model. 


\section{PeerJ Reviewing Manuscript}

\begin{tabular}{ll}
\hline Abstract component & Implementing component \\
\hline Portal & Liferay \\
Data service framework & caCORE SDK \\
\hline Meta data directory & Internal development (based on caCORE \\
& SDK) \\
Terminology server & TemaTres \\
Security service & caCORE SDK, LDAP \\
Statistics service & R \\
Document management system & Alfresco \\
Pipeline Management & Galaxy (planned) \\
\hline
\end{tabular}

